\title{
Laparoscopic totally extra peritoneal (TEP) inguinal hernia repair: A series of 47 cases
}

\section{Shrestha SK}

Suman Kumar Shrestha, Associate Professor, Department of Surgery, Kathmandu Medical College Teaching Hospital, Kathmandu, Nepal

\begin{abstract}
Background: Over the past years, several surgical techniques have been evolved, among which total extraperitoneal inguinal hernia repair is the laparoscopic technique which is more popular now.

Objective: To evaluate the outcome of totally extra peritoneal laparoscopic hernioplasty in terms of operation time, hospital stay and complications.

Methods: The prospectively designed descriptive study was carried out at Department of Surgery Unit III, Kathmandu Medical College Teaching Hospital from February 2014 to April 2015. Forty seven consecutive patients above 15 years of age underwent totally extra peritoneal repairs for inguinal hernias. The selection criteria were reducible primary or recurrent, unilateral, direct and indirect inguinal hernias. All the relevant details of each patient were noted subsequently and analyzed statistically using Statistical Program for Social Sciences (SPSS) version 15.

Results: The mean age of patient was $49 \pm 2.5$ years. The mean operation time was $65 \pm 5.3$ minutes. There were no serious complications except four (8.5\%) cases of groin seroma which resolved after single time aspiration. Three (6.3\%) cases had developed recurrence, two (4.2\%) had developed hydrocele in a median follow up period of $6 \pm 1.5$ (range, 3-9months). The mean inpatient hospital stay was $1.7 \pm 0.2$ (range, 1-2.4days).

Conclusion: Total extra peritoneal hernioplasty is safe and feasible with acceptable complications and recurrence rates.
\end{abstract}

Key words: Extra peritoneal approach, Inguinal hernia, Laparoscopic surgery,

\section{INTRODUCTION}

7 duardo Bassini published inguinal hernia repair Cin 1887.Then Shouldice repair and Lichtenstein tension free mesh repair had been popular till 1990s ${ }^{1,2}$. Several randomized controlled trials compared laparoscopic and open hernia repairs showing less operation time, reduced inpatient stay and early return to work as well as less long term complications of groin pain and paresthesia ${ }^{2,3}$. However, initial laparoscopic herniorrhaphy were associated with recurrence rates up to $25 \%$. But now with a decade of experience, recurrence rate is about $2 \% \%^{3,4}$. All myopectineal orifices of direct, indirect, femoral and obturator hernia can be inspected allowing repair of unexpected hernias. Laparoscopic hernioplasty has become the standard method for the recurrent hernias and bilateral inguinal

\section{Address for correspondence}

\section{Dr. Suman Kumar Shrestha}

Associate Professor, Department of Surgery

Kathmandu Medical College Teaching Hospital, Sinamangal, Kathmandu, Nepal

E-mail: suman_shrestha@hotmail.com hernias ${ }^{4,5}$. Minimally invasive procedures were a plug and patch repair, intra peritoneal onlay mess repair, transabdominal preperitoneal(TAPP) and totally extraperitoneal(TEP). These days most common repairs are placement of synthetic mesh in the pre peritoneal space through the abdomen (TAPP) or totally extra pre peritoneal approach (TEP) $)^{5,6}$.

In the TAPP techniques, one has to enter the peritoneal cavity leading to risks of injury to intra peritoneal contents including bladder and intestine. The laparoscopic totally extra peritoneal inguinal hernia repair (TEP) does not enter the peritoneal cavity and reduces the risk of visceral injury, adhesion formation and the development of port site hernia ${ }^{6,7}$. This study evaluates TEP hernioplasty in terms of operative time, hospital stay and complications in patients in Kathmandu Medical College Teaching Hospital.

\section{METHODS}

This prospectively designed descriptive study was carried out between February 2014 and April 2015 at the Department of Surgery Unit III in Kathmandu Medical 
College Teaching Hospital. Forty seven consecutive patients above 15 years of age underwent totally extra preperitoneal repair for inguinal hernia. Only consecutive patients with unilateral, reducible and primary inguinal hernias, both direct and indirect were included in the study. Patients with irreducible, obstructed or strangulated hernia, previous lower abdominal surgeries were excluded and they were all treated by open procedure. The patients unfit for general anesthesia were also excluded. Data on patient demography, hernia types, operative aspects, postoperative outcome and complications were recorded and subsequently analyzed using Statistical Program for Social Sciences (SPSS) version 15.

Under general anesthesia with good muscle relaxation, one centimeter longitudinal sub umbilical incision was made. Anterior rectus sheath was incised transversely and the rectus muscle was retracted laterally. Space was created between rectus muscle and peritoneum using first by gentle finger blunt dissection and introducing Hassan cannula, after which a 0 degree telescope was used to create adequate space by moving it to and fro up to the symphysis pubis. When good space was made, two $5 \mathrm{~mm}$ ports were kept in two midclavicular lines $3 \mathrm{~cm}$ below the level of umbilicus. Insufflation of this space was done by carbon dioxide $\left(\mathrm{CO}_{2}\right)$ gas at the pressure of $12 \mathrm{~mm}$ of mercury. The first step at this point was identification of all landmarks including pubic bone, Cooper ligament, spermatic cord, inferior epigastric vessels and iliac vessels. Zero degree telescope was used for better visualization in dissection. After reduction of the hernia, the second step was dissection of the sac. The indirect big sac was divided at deep ring site and peritoneal side was ligated using endo loop. The final step was to introduce $10 \times 15 \mathrm{~cm}$ polypropylene mesh through the $10 \mathrm{~mm}$ port and spread in the areas of hernial orifices from pubis in the midline to deep ring laterally. To prevent mesh migration, laparoscopic tacks were fixed at Cooper's ligament. For bilateral hernias, the same two meshes were used in the same way on both sides. Tacks were not used inferiorly to avoid injury to iliac vessels and laterally to protect iliohypogastric nerve, ilioinguinal nerve and lateral cutaneous nerve of the thigh preventing intractable pain after surgery. Finally, gas was released and port incisions were closed with absorbable sutures.

\section{RESULTS}

Altogether forty seven patients with unilateral, reducible and primary inguinal hernias underwent TEP repair. The age of the patients varied from 16 to 82 years (mean,
$49 \pm 2.5$ years). Forty two (89.3\%) were males and $5(10.6 \%)$ were females. The mean operative time for TEP repair were $65 \pm 5.3$ minutes, ranging from 25 to 105 minutes. Bilateral hernia repairs took $30 \%$ longer time than for unilateral repair(72 versus 54 minutes). The mean inpatients hospital stay was $1.7 \pm 0.2$ days ranging from one to 2.4days. The operated cases were followed up between three to nine months (median, $6 \pm 1.5$ months) (Table 1)

\section{Table 1: Patient demography and observations regarding operation time, hospital stay and follow up}

\begin{tabular}{ll}
\hline Parameters & Observations \\
\hline Number of cases & 47 \\
\hline Mean age & $49 \pm 2.5$ years (range, 16-82) \\
\hline Sex & Male 42 (89.3\%), Female 5 (10.6\%) \\
\hline Mean operation time & $\begin{array}{l}65 \pm 5.3 \text { minutes (range, } \\
25-105 \text { minutes) }\end{array}$ \\
\hline Mean hospital stay & $1.7 \pm 0.2$ days (range, 1-2.4days) \\
\hline Median follow up & $6 \pm 1.5$ months, 3-9months) \\
\hline
\end{tabular}

Out of 47 patients, 30 (63.8\%) had right sided and 17 (36.2\%) had left sided hernia. Thirty four (72.3\%) patients had direct hernias and 13 (27.6\%) had indirect hernias. (Table 2).

\section{Table 2: Characteristics of hernia $(n=47)$}

\begin{tabular}{ll}
\hline Variables & \\
Hernia sites & \\
Right & $30(63.8 \%)$ \\
Left & $17(36.2 \%)$ \\
Hernia types & \\
Direct & $34(72.3 \%)$ \\
Indirect & $13(27.6 \%)$ \\
\hline
\end{tabular}

Four patients (8.4\%) had minor complications of groin seroma formation which were aspirated and all patients recovered fully. Two patients (4.2\%) developed hydrocele. No patients had developed testicular atrophy. There was no mortality. There were three (6.3\%) recurrences. All recurrences were direct type of hernia and were managed by open surgery. In all cases we anchored mesh to Cooper's ligament in the inguinal floor (Table 3).

Table 3: Complications during and after TEP repair

\begin{tabular}{lc|}
\hline Seroma formation & $\mathbf{4 ( 8 . 5 \% )}$ \\
\hline Hydrocele & $2(4.2 \%)$ \\
\hline Recurrence & $3(6.3 \%)$ \\
\hline Testicular atrophy & 0 \\
\hline
\end{tabular}




\section{DISCUSSION}

Laparoscopic hernia repair is a comparatively new procedure in respect to open hernia repair. This technique requires expensive equipment and training in the field of laparoscopic surgery which is different from open surgery in vision, tactile sensation and dexterity. The learning curve is steep. Different literatures suggest that laparoscopic surgery in groin hernia is safe and efficacious. In addition, there is less pain, early recovery, better cosmesis and quick return to work in this approach of surgery in groin hernia. The procedure is done in day care basis in some centers but we admitted the patients for at least an overnight ${ }^{1-3}$. Nobody wanted to leave hospital without an overnight stay. We have studied 47 consecutive cases of laparoscopic hernia repair in our setup.

The results from our study are comparable to other results mentioned by many other studies which are mainly concentrated on unilateral and primary hernias. The operation time in their series was 30 to 70 minutes while recurrence rate is 1 to $6 \%$ in five years of follow up period. Similarly the complication rate of wound seroma (8.5\%) is also similar. They also mentioned the reduced overall cost in comparison to open surgery ${ }^{4-6}$.

As mentioned by other studies, for bilateral hernias TEP was easier with lower risk of injury to the intra-abdominal viscera, vessels and nerves. The same incision is enough for bilateral Stoppa mesh repair. In case of recurrent hernia, there were fewer adhesions than expected in the procedure. If patient demand laparoscopic surgery for primary unilateral hernia expecting quick recovery and return to work, as advised by National Institute of Clinical Excellence (NICE), TEP is a better choice ${ }^{7-9}$.

\section{REFERENCES}

1. Cheah WK, SO JB, Lomanto D. Endoscopic extra peritoneal inguinal hernia repair: a series of 182 repairs. Singapore Med J. 2004; 45:267-70.

2. Iuamoto LR, Kato JM, Meyer A, Blanc P. Laparoscopic totally extra peritoneal (TEP) hernioplasty using two trocars: anatomical and surgical technique. $A B C D$ Arq Bras Cir Dig. 2015; 28:121-23.

3. Shaikh AG, Soomro MI, Shaikh MS, Menon AA. Outcome of totally extra peritoneal laparoscopichernioplasty at a tertiary care hospital Larkana. J Pak Med Assoc. 2013; 63: 850-53.

4. Patel BH, Choksi DB, Vohra AS. Evaluation of cases of laparoscopic inguinal hernia repairat government medical college Vadodara. Int J Res Med.2014; 3:6671.
Intraoperative complications are rare but bladder injury in $0.2 \%$, bowel injury in $0.6 \%$ and fatal vessel injury in $0.1 \%$ are recorded. Bladder catheterization, Trendelenberg position, avoid stapling in lower and lateral aspects and following spider web aspects in dissection prevent the dangerous complications. However, we did not have such complications as the number of cases was still small. TEP hernioplasty is an advanced laparoscopic procedure and relative contraindications should be kept in mind. They are patients unfit for general anesthesia, obesity, larger hernia, pregnant woman, history of lower abdominal surgery, recurrent laparoscopic hernia and patients receiving anticoagulants. A learning curve of 40 cases in high flow center is necessary to avoid complications and recurrences ${ }^{10-12}$.

Postoperative hospital stay was $1.7 \pm 0.2$ days (range, 1-2.4days) which is comparable with study done by Awdal ZA et al which was $1.52 \pm 0.51$ days. This is more in open hernia repair group which is $2.24 \pm 0.97$ days. There were no cardiopulmonary, cerebrovascular and thrombotic complications in our study and in the study done by Picazo. Cases of hernias with patent processus vaginalis in boys aged 16, were treated by herniotomy at deep ring by z-suture alone in other studies. However, we performed it by formal TEP and mesh fixation ${ }^{13-16}$.

\section{CONCLUSION}

Laparoscopic extraperitoneal hernia repair is appropriate for patients as an alternative to open hernia surgery. It is safe and effective method for cases of inguinal hernia with advantage of less operative time, less post-operative stay, with acceptable complications and recurrence rate.

5. SrivastavaA, Singh R, Pal LS. A comparative study in laparoscopic inguinal hernia repair betweenfixations versus non fixation of mesh. J. Evid. Based. Med. Healthc. 2016; 3:490-92.

6. Fegade S. Laparoscopic versus open repair of inguinal hernia. World Journal of Laparoscopic Surgery.2008;1:41-48.

7. Jain SK, Gupta A, Kumar S,Kaza RCM. Laparoscopic versus open inguinal hernia repair: A Systematic Review of Literature. Asian Journal of Medical Sciences. 2014;5:10-14.

8. Kavic SM. Laparoscopic versus open repair: A superior approach to inguinal herniorrhaphy. OA Minimally Invasive Surgery. 2013;1: 1-4. 
9. Feliu X, Claveria R, Besora P, Fernandez-Sallent E et al. Bilateral inguinal hernia repair:Laparoscopic or open approach. Hernia. 2011;15:15-18.

10. Meyer AL, BellandiDM,DelacosteFetal.Laparoscopic totally extra peritoneal inguinal hernia repair:Non fixation of three dimensionalmesh. Brazilian Journal of Video Endoscopic Surgery. 2010;1:19-23.

11. Meyer A, Dulucq JL, Mahajna A. Laparoscopic hernia repair: Non fixation mesh feasibility.ABCD Arq Bras Cir Dig. 2013;26:27-30.

12. Ciftci F. Laparoscopic versus open inguinal hernia Repair on patients over 75 years of age. Int J ClinExp Med. 2015; 8: 10016-20.

13. Shalaby RY, Fawy M, Soliman SM, Dorgham A. Needlescopicherniorrhaphy in children - a simplified technique. J Indian AssocPediatr Surg. 2005;10:168-71.

14. Nawaz A, Mansoor R, Butt Ul et al. Comparison of laparoscopic total extra peritoneal repair with Lichtenstein repair in inguinal hernia.Journal of Surgery Pakistan International. 2015; 20: 40-43.

15. Kumar A, Ramakrishnan T. Single port laparoscopic repair of pediatric inguinal hernias: our experience at a secondary care centre. Journal of Minimal Access Surgery. 2013; 9:7-12.

16. Panse M, Despande N, Mandhane A, Bhalerao P. Seroma prevention technique following endoscopic direct inguinal hernia repair. Journal of Evolutionof Medical and Dental Sciences.2013; 2; 4928-32. 